\title{
Um Plano de Testes para Apoiar o Processo de Avaliação de Acessibilidade de uma Página Web segundo as WCAG 2.0
}

\author{
Leonardo L. Nardi, Carolina C. Sacramento, Maria Alice S. Brito \\ Instituto de Matemática e Estatística - Departamento de Informática e Ciência da \\ Computação - Universidade do Estado do Rio de Janeiro (UERJ) \\ dsnardi@gmail.com, carolinasacramento@globo.com, malice@ime.uerj.br
}

\begin{abstract}
Resumo. Este artigo descreve um plano de testes para auxiliar a avaliação de acessibilidade de páginas e sítios na Web. O plano é uma extensão do planejamento de avaliação de acessibilidade inerente aos critérios de sucesso das WCAG 2.0. Essa avaliação é reconhecidamente complexa e volumosa. Além disso, os resultados desse processo apenas indicam os itens que não estão em conformidade com os critérios de sucesso, sem sugerir soluções. Sendo assim, o plano de testes apresentado neste trabalho traz duas contribuições: i) ordena as etapas de avaliação; e ii) inclui no relatório final as sugestões de correção para cada falha.

Palavras-chave: Acessibilidade na Web, Avaliação de acessibilidade na Web.

Abstract. This paper describes a test plan to support the accessibility evaluation of Web pages and sites. This plan is an extension of the accessibility evaluation planning inherent to WCAG 2.0 success criteria. This evaluation is admittedly complex and extensive. In addition, the results indicate only items that do not conform to the success criteria, without recommending solutions. Thus, the test plan presented in this work brings two contributions: i) sorts the evaluation steps and ii) includes suggestions to repair each failure in the final report.
\end{abstract}

Keywords: Web accessibility, Web accessibility evaluation

\section{Introdução}

As possibilidades trazidas pela Internet, tais como compartilhamento de dados, comunicação instantânea, aquisição de produtos e serviços, e acesso a informações em ambiente virtual e em escala mundial, permitindo interatividade, levaram à participação social em grande escala sem limites geográficos.

No mundo real, tanto os ambientes circulados e produtos utilizados/consumidos, quanto bibliotecas, restaurantes, universidades, automóveis, por exemplo, precisam ser arquitetados e construídos com base nos conceitos do desenho universal e da acessibilidade. Esses mesmos conceitos são necessários também no mundo virtual, a Internet.

A acessibilidade na $W e b$ é um tema rico e ao mesmo tempo premente, trazendo a chance da inclusão digital aos portadores de necessidades especiais. Assim sendo, abrese, a essas pessoas, a oportunidade de realizar, confortavelmente, as operações 
disponíveis na $W e b$, sejam elas de serviços, comerciais, acadêmicas, entretenimento, dentre outras.

Esse tema tem sido alvo de interesses, com pesquisa e desenvolvimento [Lawrence and Giles 2000, Lazar et al. 2004, Henry 2006, Henry 2006, CRPD 2008, W3C-a], além do estabelecimento de recomendações, há mais de uma década. Em 1999, foi lançada a primeira versão da Web Content Accessibility Guidelines (Guia de Recomendações para Acessibilidade na Web) WCAG 1.0 [W3C-b] e em 2008 a última versão, denominada WCAG 2.0 [W3C-c].

Mesmo com esses esforços para a definição de recomendações voltadas para a acessibilidade na $W e b$, o que é apresentado na seção 2, a maioria das páginas e sítios Web não atende a essas recomendações. Essa lacuna é a razão principal deste trabalho, que se situa na avaliação de acessibilidade de páginas e sítios Web.

As WCAG 2.0 foram estabelecidas com dois objetivos principais, a testabilidade e a independência quanto a evoluções tecnológicas. Os critérios de sucesso distribuídos pelas diretrizes e princípios das WCAG 2.0 podem ser aplicados como um checklist na avaliação de acessibilidade de uma página ou sítio na Web [Fernandes et al. 2011]. Esse processo, habitualmente, é realizado em duas etapas de validação: automática e manual. Uma avaliação desse tipo gera um relatório que aponta os itens que não estão em conformidade com os critérios de sucesso.

O World Wide Web Consortium (W3C) [W3C-a] recomenda que os avaliadores de acessibilidade sejam muito preparados quanto ao domínio de tecnologias $W e b$, das ferramentas de avaliação, das barreiras que impedem a acessibilidade, das tecnologias assistivas, bem como das próprias recomendações WCAG 2.0, em face da complexidade envolvida nessa avaliação.

No processo de avaliação, para a validação automática, há diversas ferramentas disponíveis no sítio do World Wide Web Consortium (W3C) [W3C-d]. Além dessa disponibilidade, o W3C também orienta a escolha da ferramenta mais apropriada à página ou ao sítio que está para ser avaliado.

Para a validação manual (visual e auditiva), há ferramentas que possibilitam o registro das falhas e das indicações de suas correções.

A validação manual (visual e auditiva) consiste em uma varredura por todos os itens da página, segundo os critérios de sucesso das WCAG 2.0. Esse processo costuma resultar em um número considerável de problemas que a validação automática não identifica [Koutsabasis et al. 2010]. Diante dessa complexidade e volume, esses autores, além de concordar com o W3C sobre a recomendação de que o avaliador possua preparo, sugerem a inclusão de organização e emprego de técnicas, na gerência da avaliação. Em [Lang 2003], essas sugestões também são consideradas, devendo ainda ser acrescentada a descrição das soluções para as falhas, no relatório final da avaliação.

Essas sugestões apontadas, em [Koutsabasis et al. 2010, Lang 2003], foram adotadas como extensão do planejamento intrínseco às WCAG 2.0, resultando na especificação do plano de testes, apresentado na seção 4, sendo a contribuição deste trabalho. 
$\mathrm{Na}$ experiência de avaliação de uma página desenvolvida sem o compromisso com a acessibilidade, foi empregado o plano de testes do presente trabalho. O nível de conformidade adotado nessa avaliação foi o AAA [W3C-c], considerado o mais rigoroso. Por tratar-se de uma página simples, apenas 51\% dos critérios de sucesso aplicaram-se, nos quais pouco mais da metade apresentaram falhas. Essas falhas detectadas foram apontadas no relatório, acompanhadas das sugestões para as suas correções, contribuindo para que o responsável pelos seus reparos faça uso desta orientação.

Outra questão que surge nas experiências com avaliação de acessibilidade aplicada por diferentes avaliadores a uma mesma página ou sítio é a produção de resultados diferentes, conforme observações apontadas na seção 3, de trabalhos relacionados.

\section{Acessibilidade na $W e b$}

A acessibilidade em seu termo mais amplo é uma qualidade diretamente relacionada ao conceito de desenho universal, pois, são seus princípios que estabelecem os requisitos de acessibilidade de quaisquer produtos [Gil 2006]. Este termo, desenho universal, foi definido, em 1997, por Ronald L. Mace [UDI-a], como o "design de produtos e ambientes a serem usados por todas as pessoas, na maior medida possível, sem a necessidade de adaptação ou desenho especializado”. Para reforçar a prática deste conceito, um grupo de arquitetos sob a liderança deste autor, Ronald L. Mace, estabeleceu os sete princípios do desenho universal, que vêm sendo adotados em todo o mundo [UDI-b], como a seguir: "1: Uso equitativo. O design é útil e comercializável às pessoas com habilidades diversas; 2: Flexibilidade em uso. O design acomoda uma ampla variedade de preferências e habilidades individuais; 3: Uso simples e intuitivo. Uso do design é fácil de entender, independentemente da experiência do utilizador, conhecimentos, habilidades de linguagem, ou nível de concentração corrente; 4: Informação perceptível. O design comunica eficazmente a informação necessária para o usuário, independentemente das condições ambientais ou habilidades sensoriais do usuário; 5: Tolerância de erro. O design minimiza perigos e consequências adversas de ações acidentais ou não intencionais; 6: Baixo esforço físico. O projeto pode ser usado de forma eficiente e confortável e com um mínimo de fadiga; 7: Tamanho e espaço para $o$ acesso e uso. Tamanho apropriado e um espaço para abordagem, alcance, manipulação e uso independentemente do tamanho do corpo do usuário, postura ou mobilidade".

No contexto da $W e b$, a acessibilidade é a propriedade que uma página ou sítio Web possui de permitir que pessoas com alguma deficiência possam usá-la com a mesma eficácia que as demais [Slatin and Rush 2003]. Em [Brajnick et al. 2010], a definição de acessibilidade na Web é estendida, abrangendo os aspectos técnicos da interface de usuário e a maneira como as pessoas percebem, interpretam e agem com essa interface.

O impedimento de acesso às pessoas com algum tipo de deficiência ocorre devido a barreiras, que aparecem evidenciadas no objetivo principal da Convenção sobre os Direitos das Pessoas com Deficiência das Nações Unidas, que foi publicada desde 2008 [CRPD 2008], reproduzido, a seguir: "promover, proteger e assegurar o 
desfrute pleno e equitativo de todos os direitos humanos e liberdades fundamentais por parte de todas as pessoas com deficiencia e promover o respeito pela sua inerente dignidade. Pessoas com deficiência são aquelas que têm impedimentos de natureza física, intelectual ou sensorial, os quais, em interação com diversas barreiras, podem ter obstruída a sua participação plena e efetiva na sociedade com as demais pessoas". Mais, especificamente, no artigo $9^{\circ}$ dessa convenção, intitulado "Acessibilidade", são enumerados os objetos físicos ou de serviços, cujo acesso deve ser assegurado às pessoas com deficiências, em igualdade de oportunidades com as demais e por essa razão devem ser submetidos à identificação e à eliminação de barreiras. Desse elenco, fazem parte os sistemas e tecnologias da informação e comunicação.

Tim Berners [W3C-e] chama a atenção para o compromisso fundamental, desde a criação da $W e b$, com a acessibilidade a todas as pessoas, independentemente, do seu hardware, software, língua, cultura, localização, capacidade física ou mental. $\mathrm{Na}$ sua concepção, se uma aplicação Web desenvolvida alcança esse objetivo, ela é acessível às pessoas de uma faixa diversa de audição, movimento, visão e capacidade cognitiva. Isso faz com que o impacto da deficiência seja alterado, radicalmente, removendo barreiras de comunicação e interação, que as pessoas com deficiência encontram no mundo físico. A justificativa do autor para o surgimento de barreiras em sítios ou páginas é a de que seus sistemas foram mal concebidos e desenvolvidos, causando, assim, a exclusão de pessoas de seu uso.

Entre as iniciativas para conduzir a $W e b$ ao seu potencial, para que se torne acessível, permitindo que as pessoas com deficiência participem do mesmo modo que as demais, foi articulada a criação do comitê gestor Web Accessibility Initiative (WAI) [W3C-f]. Com esse compromisso, a função do departamento de acessibilidade, cujo nome coincide com o do comitê, tem a função de desenvolver padrões abertos, que garantam o crescimento, a longo prazo, elaborando estratégias, diretrizes e recursos para que as pessoas com deficiência participem igualmente na Web. Os autores, em [Dias 2007, Melo et al. 2004], consideram ainda a flexibilidade proporcionada pela $W e b$, para que seu uso seja permitido em diferentes ambientes e situações, e através de vários equipamentos e navegadores.

Uma referência a ser adotada no desenvolvimento de aplicações Web tal como a dos princípios para desenho universal fazia-se necessária. Assim, em outubro de 1994, cinco anos após a criação da World Wide Web, seu inventor, Tim Berners, fundou o World Wide Web Consortium (W3C) [W3C-a].

Em maio de 1999, foi publicada, pelo WAI/W3C, a primeira versão do guia $\mathrm{Web}$ Content Accessibility Guidelines (WCAG1.0) [W3C-b]. Nove anos depois, em dezembro de 2008, era lançada a segunda versão desse guia, a WCAG 2.0 [W3C-c], baseada na versão anterior e concebida para ser aplicada em larga escala a diferentes tecnologias Web, atuais e futuras, e ser testável com uma combinação de testes automáticos e avaliação manual.

Essas recomendações de acessibilidade para o conteúdo da Web (WCAG 2.0) definem a forma como tornar esse conteúdo mais acessível a pessoas com deficiências. Elas foram desenvolvidas em colaboração mundial, com o objetivo de elaborar um padrão compartilhado referente à acessibilidade, que satisfaça às necessidades das pessoas com deficiência, das organizações e dos governos, em nível internacional 
[Bechara 2009]. Dessa forma, consideraram uma vasta gama de deficiências, como a seguir: i) Cegueira, daltonismo e baixa visão; ii) Surdez e baixa audição; iii) Dificuldade de aprendizagem; iv) Limitações cognitivas; v) Limitações de movimento; vi) Incapacidade de fala; vii) Fotossensibilidade e suas combinações; viii) Dificuldades adquiridas no processo de envelhecimento; ix) Barreiras territoriais e sociais. Embora a abrangência dessas recomendações definidas pelo W3C seja bem ampla, ainda há necessidades de pessoas com outros tipos, graus e combinações de deficiências que não estão abordadas nas WCAG 2.0 [Bechara 2009]. Cabe notar que a finalidade principal das recomendações de acessibilidade é atender pessoas com deficiências, contudo a sua aplicação traz benefícios aos usuários em geral.

A acessibilidade na $W e b$ depende não só do conteúdo acessível, como também da capacidade de leitura desse mesmo conteúdo pelos navegadores e outros agentes do usuário, que são quaisquer softwares que obtenham e apresentem conteúdos da Web aos usuários, incluindo as tecnologias assistivas. Este termo é a tradução de Assistive Technology, que foi concebido, em 1988, por ocasião do estabelecimento de um conjunto de leis norte-americanas (Public Law 10-407). Este conjunto aliado ao American with Disabilities Act (ADA) estabelece os direitos dos cidadãos norteamericanos com necessidades especiais, além de regulamentar a comercialização com dinheiro público de recursos para atendê-los. Por conceito, tecnologia assistiva se refere a "qualquer item, peça de equipamento ou produto, adquirido, modificado ou personalizado para ampliar, manter ou melhorar as capacidades funcionais de indivíduos com deficiência" [IDEA], tendo esses itens sido classificados em onze categorias, como a saber: i) Auxílios para a vida diária; ii) CAA (CSA) - Comunicação aumentativa (suplementar) e alternativa; iii) Recursos de acessibilidade ao computador; iv) Sistemas de controle de ambiente; v) Projetos arquitetônicos para acessibilidade; vi) Órteses e próteses; vii) Adequação Postural; viii) Auxílios de mobilidade; ix) Auxílios para cegos ou com visão sub-normal; $\mathrm{x}$ ) Auxílios para surdos ou com déficit auditivo; e xii) Adaptações em veículos.

No que tange à acessibilidade na $W e b$, as tecnologias assistivas relacionadas são as que pertencem à categoria "Recurso de Acessibilidade ao computador": equipamentos de entrada e saída (síntese de voz, Braille), auxílios alternativos de acesso (ponteiras de cabeça, de luz), teclados modificados ou alternativos, acionadores, softwares especiais (de reconhecimento de voz, etc.), que permitem às pessoas com deficiência o uso do computador. Entre as tecnologias assistivas desta categoria, destacam-se o sistema operacional DOSVOX [DOSVOX], e os softwares leitores de tela Jaws for Windows [JAWS], Virtual Vision [Virtual Vision] e NVDA - NonVisual Desktop Access [NV Access]. Esses aplicativos possuem sintetizadores de voz, que associam códigos de páginas Web em voz sintetizada ou gravada previamente e possibilitam a integração do defíciente visual à Internet.

No contexto deste trabalho, dirigido a pessoas com deficiência(s), é interessante o uso do aplicativo Webvox, navegador Web do sistema operacional DOSVOX, em razão de ser uma tecnologia de apoio bastante difundida nas universidades brasileiras e no Instituto Benjamin Constant (IBC) para inclusão social, tecnológica e intelectual do indivíduo. Além disso, esse software é gratuito e de fácil uso. Segundo o co-criador do sistema, professor Antônio Borges do NCE/UFRJ o número de usuários deste sistema, em 2011, chegava a 30.000, em toda a América Latina. Este sistema também costuma 
ser usado algumas vezes combinado a outras tecnologias baseadas na síntese de fala, na maior parte das instituições de cegos, o que inclui o IBC.

Para perfeito funcionamento das tecnologias assistivas mencionadas anteriormente, é fundamental que os sítios e portais tenham sua construção bem estruturada no que diz respeito à aplicação correta das linguagens de marcação (HTML, XHTML) e das folhas de estilo em cascata (CSS), em sua semântica e sintaxe, de forma que seus códigos sejam lidos e sintetizados em voz pelos leitores de tela adequadamente. Esses requisitos, aliados ao atendimento das recomendações de acessibilidade do WAI, as WCAG2.0, garantem ou pelo menos prometem garantir maior integração deste público no ambiente virtual.

A acessibilidade na $W e b$, no Brasil, é regulamentada por decreto. Em 02 de dezembro de 2004, o então presidente Luís Inácio Lula da Silva, tornou público o

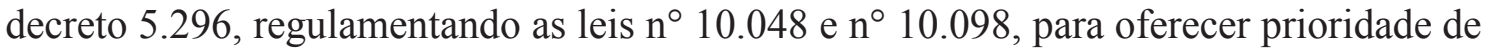
atendimento às pessoas beneficiadas por estas duas leis e estabelecer normas gerais e critérios básicos para a promoção da acessibilidade das pessoas portadoras de deficiência ou com mobilidade reduzida, entre outras providências [Decreto 5.296]. Particularmente, no capítulo VI deste decreto, é tratado o acesso à informação e à comunicação, regulamentando o acesso pleno, nos portais e sítios eletrônicos da administração pública, a portadores de deficiência visual. Embora a legislação obrigue a observância da acessibilidade na construção de portais, sítios, e páginas $W e b$ do governo, um estudo sobre as dimensões e características da Web brasileira (Censo web.br [Vieira 2010]), realizado pelo Comitê Gestor da Internet no Brasil, em 2010, demonstrou, entre outras constatações, que apesar de existir um decreto regulamentando a acessibilidade, conforme figura 1 , apenas $2 \%$ dos sites pesquisados sob o domínio "gov.br" apresentavam algum tipo de conformidade com os padrões de acessibilidade do governo federal, enunciados no Modelo de Acessibilidade do Governo Eletrônico (e$\mathrm{MAG})$ [e-MAG].

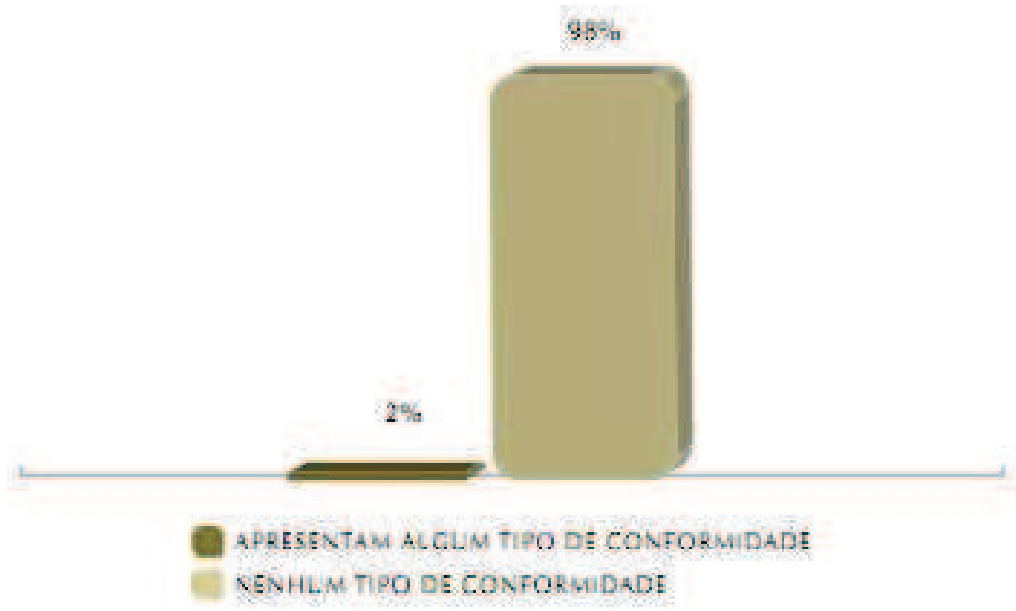

Fig. 1. Proporção de páginas sob domínio "gov.br" aderentes aos padrões de acessibilidade [Vieira 2010]

O e-MAG é representado por um conjunto de recomendações a ser considerado para que o processo de acessibilidade dos sítios e portais do governo brasileiro seja conduzido de forma padronizada e de fácil implementação. Ele é uma adaptação de padrões internacionais às necessidades brasileiras e foi formulado para orientar 
profissionais que tenham contato com publicação de informações ou serviços na Internet a desenvolver, alterar e/ou adequar páginas, sítios e portais, tornando-os acessíveis ao maior número de pessoas possível [e-MAG].

Em 07 de maio de 2007, o governo institucionalizou o e-MAG por meio da portaria número três, tornando sua observância obrigatória nos sítios e portais governamentais. Embora, tenha sido institucionalizado esse conjunto de recomendações do governo federal, a sua versão disponível ainda encontra-se baseada nas WCAG 1.0. A abordagem deste trabalho adotou a versão mais recente das recomendações WCAG, a versão 2.0 [W3C-c].

\subsection{Estrutura e organização das WCAG 2.0}

As recomendações WCAG2.0 são organizadas por quatro princípios: Perceptível, Operável, Compreensível e Robusto. Cada princípio, por sua vez, contém diretrizes e critérios de sucesso. Esses critérios de sucesso permitem testar a acessibilidade das páginas web. Caso o resultado dos testes esteja em conformidade com esses critérios de sucesso é garantido ao usuário, com algum tipo de deficiênciaa, fazer uso das informações na $\mathrm{Web}$ sem grandes dificuldades.

Neste ponto, foi considerado apropriado reproduzir trechos das WCAG 2.0, encontradas traduzidas [WCAG 2.0-pt], como a seguir:

"Noções sobre os Quatro Princípios de Acessibilidade

As directrizes e os Critérios de Sucesso estão organizados em torno de quatro princípios, que apresentam a informação básica para um utilizador aceder e utilizar os conteúdos da Web. Um utilizador que pretenda utilizar a Web tem de dispor de conteúdo que seja:

1. Perceptivel - A informação e os componentes da interface de utilizador têm de ser apresentados aos utilizadores de formas perceptiveis.

- Isto significa que os utilizadores têm de ser capazes de compreender a informação apresentada (tem de estar visivel a todos os seus sentidos)

2. Operável - Os componentes da interface de utilizador e a navegação têm de ser operáveis.

- Isto significa que os utilizadores têm de ser capazes de funcionar com a interface (a interface não pode requerer uma interacção que um utilizador não possa executar)

3. Compreensivel - A informação e a operação da interface de utilizador têm de ser compreensíveis.

- Isto significa que os utilizadores têm de ser capazes de compreender a informação e o modo de funcionamento da interface de utilizador (os conteúdos ou o funcionamento não podem ir para além da sua compreensão)

4. Robusto - O conteúdo tem de ser suficientemente robusto para ser interpretado, com precisão, por uma grande variedade de agentes de utilizador, incluindo tecnologias de apoio. 
- $\quad$ Isto significa que os utilizadores têm de ser capazes de aceder aos conteúdos à medida que as tecnologias avançam (à medida que as tecnologias e os agentes de utilizador evoluem, os conteúdos devem permanecer acessiveis)

Se algum destes princípios não for verdadeiro, os utilizadores com incapacidades não serão capazes de utilizar a Web.

A seguir a cada princípio, são apresentadas directrizes e Critérios de Sucesso para ajudar as pessoas com incapacidades a abordar os princípios. Existem muitas diretrizes de utilização geral que tornam os conteúdos mais utilizáveis por todos os utilizadores, incluindo aqueles com incapacidades. Contudo, nas WCAG 2.0 apenas estão incluidas as directrizes que abordam problemas especificos de pessoas com incapacidades. Isto inclui problemas que bloqueiam o acesso ou que interferem, de forma mais séria, com o acesso à Web, por parte das pessoas com incapacidades."

As diretrizes são definidas como uma generalização que informa aos desenvolvedores, no âmbito geral, como deve ser tratada e aplicada a acessibilidade no contexto da informação a ser apresentada ao utilizador.

O objetivo das diretrizes é garantir que os conteúdos estejam acessíveis ao maior número possível de utilizadores, de modo a atender às diferentes deficiências. Sejam elas: sensoriais, físicas ou cognitivas.

Para cada diretriz, são fornecidos critérios de sucesso testáveis que determinam de forma objetiva se os conteúdos oferecidos pelos portais, sítios e páginas satisfazem às recomendações, ou seja, como deve ser apresentada uma dada informação ao utilizador.

Há níveis de acessibilidade, denominados níveis de conformidade, a serem alcançados pelos portais, sítios e páginas que vão desde uma acessibilidade restrita ao extremo de atender a todas as recomendações das WCAG 2.0. Esses níveis, dependendo da finalidade informativa de cada portal, sítio ou página, e para qual público alvo é destinado, são designados em: nível A (o mais baixo), nível AA e nível AAA (o mais alto).

Para dar uma noção desses níveis de conformidade, foi trazida uma tradução da WCAG 2.0 [WCAG 2.0-pt], como a seguir:

"Noções sobre os Niveis de Conformidade

Primeiro, existe uma série de condições que têm de ser cumpridas para que um Critério de Sucesso seja incluído. Estas incluem:

i. Todos os Critérios de Sucesso têm de ser problemas de acesso importantes para as pessoas com incapacidades que abordam problemas que ultrapassam os problemas de utilização que podem ser enfrentados por todos os utilizadores. Por outras palavras, o problema de acesso tem de originar um problema proporcionalmente maior para pessoas com incapacidades do que para pessoas sem incapacidades para ser considerado um problema de acessibilidade (e abrangido por estas directrizes de acessibilidade). 
ii. Todos os Critérios de Sucesso têm igualmente de ser testáveis. Isto é importante, uma vez que, de outra forma, não seria possivel determinar se uma página cumpriu ou falhou no cumprimento dos Critérios de Sucesso. Os Critérios de Sucesso podem ser testados através de uma combinação de avaliação automática e humana, desde que seja possível determinar se um Critério de Sucesso foi cumprido com um elevado nível de confiança.

O grupo de trabalho atribuiu um dos três níveis de conformidade a cada Critério de Sucesso depois de levar em consideração uma vasta gama de problemas de interação. Alguns dos fatores comuns avaliados ao definir o nível incluem:

- se o Critério de Sucesso é essencial (por outras palavras, se o Critério de Sucesso não for cumprido, nem a tecnologia de apoio pode tornar o conteúdo acessivel)

- se é possivel cumprir o Critério de Sucesso para todos os sitios da Web e tipos de conteúdo aos quais se aplicam os Critérios de Sucesso (por ex., diferentes tópicos, tipos de conteúdo, tipos de tecnologia Web)

- se o Critério de Sucesso necessita de conhecimentos que podem ser razoavelmente alcançados pelos criadores do conteúdo (ou seja, o conhecimento e a capacidade para cumprir os Critérios de Sucesso podem ser alcançados numa semana de formação ou menos)

- $\quad$ se o Critério de Sucesso impõe limites no "aspecto" elou funcionamento da página Web (limites no funcionamento, apresentação, liberdade de expressão, design ou estética que os Critérios de Sucesso poderão colocar aos autores).

- $\quad$ se não existem soluções no caso de os Critérios de Sucesso não serem cumpridos".

Os critérios de sucesso são testáveis e determinam de forma objetiva se um conteúdo cumpre toda uma especificação. Essa condição de ser testável permite que esses critérios distribuídos pelas diretrizes e princípios da WCAG possam ser aplicados como um checklist na avaliação de acessibilidade de uma página, sítio, ou portal, na Web [Fernandes et al. 2011].

\subsection{Checklist}

Neste trabalho, foi utilizado o checklist fornecido pela WebAIM [WebAIM], uma empresa sem fins lucrativos que objetivou facilitar os testes de acessibilidade com base nos critérios de sucesso das WCAG 2.0. Sua estrutura é distribuída em princípios, diretrizes e critérios, de acordo com a hierarquia encontrada nessas recomendações.

Essa estrutura é formada por quatro listas principais representando os princípios. Cada lista associada a um princípio contém por sua vez listas representando as diretrizes desse princípio. Cada lista associada a uma diretriz por sua vez contém os elementos que representam os critérios de sucesso. Cabe notar que algumas vezes um critério de sucesso é representado por mais de um elemento da lista. Cada elemento no checklist possui três campos: i) a identificação do critério de sucesso análogo à identificação que consta nas recomendações da WCAG 2.0; ii) um campo para indicar se o critério de 
sucesso foi correspondido; e iii) texto com a descrição encontrada nas recomendações para o critério de sucesso em questão.

A figura 2 mostra como exemplo apenas uma lista, que representa a diretriz " 3.2 Previsível" do princípio "3. Compreensível". O guia completo encontra-se reproduzido em [Nardi and Sacramento 2011].

Guideline 3.2

Predictable: Make Web pages appear and operate in predictable ways

\begin{tabular}{|c|c|}
\hline $\begin{array}{l}\text { Success } \\
\text { Criteria }\end{array}$ & WebAIM's Recommendations \\
\hline$\frac{3.2 .1 \text { On }}{\text { Focus }}$ & $\begin{array}{l}\text { When a page element receives focus, it does not result in a } \\
\text { substantial change to the page, the spawning of a pop-up window, } \\
\text { an additional change of keyboard focus, or any other change that } \\
\text { could confuse or disorient the user. }\end{array}$ \\
\hline$\frac{3.2 .20 n}{\text { Input }}$ & $\begin{array}{l}\text { When a user inputs information or interacts with a control, it does } \\
\text { not result in a substantial change to the page, the spawning of a } \\
\text { pop-up window, an additional change of keyboard focus, or any other } \\
\text { change that could confuse or disorient the user unless the user is } \\
\text { informed of the change ahead of time. }\end{array}$ \\
\hline$\frac{\frac{3.2 .3}{\text { Consistent }}}{\frac{\text { Navigation }}{\text { (Level AA) }}}$ & $\begin{array}{l}\text { Navigation links that are repeated on web pages do not change order } \\
\text { when navigating through the site. }\end{array}$ \\
\hline \begin{tabular}{l|}
$\frac{3.2 .4}{\text { Consistent }}$ \\
Identification \\
(Level AA)
\end{tabular} & $\begin{array}{l}\text { Elements that have the same functionality across multiple web pages } \\
\text { are consistently identified. For example, a search box at the top of } \\
\text { the site should always be labeled the same way. }\end{array}$ \\
\hline \begin{tabular}{l|}
$\frac{3.2 .5 \text { Change }}{\text { on Request }}$ \\
(Level AAA)
\end{tabular} & $\begin{array}{l}\text { Substantial changes to the page, the spawning of pop-up windows, } \\
\text { uncontrolled changes of keyboard focus, or any other change that } \\
\text { could confuse or disorient the user must be initiated by the user. } \\
\text { Alternatively, the user is provided an option to disable such changes. }\end{array}$ \\
\hline
\end{tabular}

Figura 2. Trecho do checklist que corresponde à diretriz 3.2.

\subsection{Considerações}

A incorporação da acessibilidade a uma aplicação Web, durante o seu desenvolvimento, atenua a quantidade de erros encontrados durante os testes de acessibilidade. A tarefa de avaliação de acessibilidade, na etapa de validação manual é complexa, devido à varredura pelos itens, verificando sua conformidade com os critérios de sucesso. Quando surge um grande número de itens que não estão em conformidade com os critérios de sucesso estabelecidos, a complexidade aumenta ainda mais. Constatada essa dificuldade, as ações no planejamento inerente às WCAG 2.0 foram combinadas a mais duas extensões, concebendo um plano de testes, para auxiliar a gerência desse processo de avaliação e a consequente tarefa de reparo, que pode ser visto na seção 4, mais à frente. 


\section{Trabalhos relacionados}

Os três trabalhos, a seguir, abordam a testabilidade dos critérios de sucesso das WCAG 2.0, ao mesmo tempo que explicitam a dificuldade de realizar uma avaliação de acessibilidade na $W e b$, até mesmo por avaliadores preparados : a) a pesquisa sobre avaliação de acessibilidade, descrita em [Brajnick et al. 2010], foi desenvolvida com avaliadores com e sem experiência. Seus resultados revelaram que até mesmo avaliadores experientes apontaram falsos positivos e falsos negativos, além de não perceberem alguns verdadeiros problemas. Os autores, nesse trabalho, admitem que a avaliação analítica (manual) pode ser influenciada por decisões subjetivas dos avaliadores, mesmo que na formulação dos critérios de sucesso da WCAG 2.0 tenha sido enfatizada a sua testabilidade; b) uma outra experiência encontra-se relatada em [Alonso et al. 2010]. Dela participaram 17 avaliadores iniciantes, que realizaram a avaliação somente manual, com nível de conformidade A, com 25 critérios de sucesso a serem avaliados. A opção pela avaliação somente manual foi justificada pela inexistência no momento de uma ferramenta automática. Um dos resultados dessa experiência mostrou que houve concordância somente em 11 dos 25 critérios de sucesso para somente 14 dos 25 alunos. Os dois instrutores também avaliaram e somente concordaram com 13 dos 25 critérios de sucesso, mas depois discutindo esses resultados, chegaram a um consenso. Esse problema de diferenças entre as avaliações de uma mesma página tem sido alertado no contexto de avaliação de acessibilidade. Outra constatação feita pelos autores nessa experiência foi a da dificuldade que iniciantes tiveram com a linguagem e os conceitos usados nos critérios de sucesso das WCAG 2.0; c) essa terceira experiência [Koutsabasis et al. 2010] de avaliação ainda foi baseada nas WCAG 1.0 e serviu para o estudo dos problemas em acessibilidade na Web. Suas conclusões permitiram aos autores determinar três passos importantes a serem encaminhados por uma gerência de avaliação de acessibilidade na $W e b$, baseada nas WCAG 2.0: (i) a identificação das necessidades dos usuários para estabelecer metas de acessibilidade; (ii) o processo da avaliação da acessibilidade, dividido nas duas validações: automática e manual, além da recomendação do teste de campo por usuários. Neste item, é confirmada a necessidade de avaliadores preparados, requerida pelo W3C [W3C-a], para avaliações de acordo com as WCAG 2.0 [W3C-c]. Além dessa confirmação, ainda é aconselhada a inclusão de organização e emprego de técnicas para apoiar o trabalho da gerência de avaliação, devido ao número considerável de problemas que surgem na avaliação manual; e (iii) definição e acompanhamento de uma política de acessibilidade. Os autores em [Koutsabasis et al. 2010] lamentam a ausência de uma ferramenta de avaliação de acessibilidade residente em servidores $W e b$.

Outra crítica encontrada é sobre a abordagem do uso de diretrizes de propósito geral, sem a flexibilidade para uma avaliação particular, encontrada em metodologias existentes. Cabe notar que essa questão foi tratada também no item (i) da metodologia proposta, em [Koutsabasis et al. 2010], descrita no grupo de trabalhos anterior. Os outros trabalhos que trazem essa crítica são os seguintes: a) uma metodologia desenvolvida para facilitar a tarefa de avaliação, dirigida a iniciantes, chamada de Accessibility Evaluation Assistant (AEA) [Bailey and Pearson 2010], tem como objetivo funcionar como uma ferramenta de suporte educacional, permitindo ser adaptada a tipos específicos de aplicação, ou grupos de usuários, ou dispositivos de acesso. Essa 
adaptação pode dar prioridade a verificações pertinentes ao tipo da aplicação, reduzindo os esforços com uma avaliação; b) um outro trabalho relatado em [Gay and Qi Li 2010] é a ferramenta AChecker, que também oferece flexibilidade, provendo interação e configuração para a avaliação pretendida. A motivação para esse trabalho também foi o problema enfrentado nas avaliações, em que diferentes resultados são encontrados, quando diferentes avaliadores até mesmo experientes realizam avaliações de uma mesma aplicação e utilizam a mesma ferramenta. Para evitar essa diferença de resultados, essa ferramenta apresenta transparência durante a avaliação, permitindo que seus usuários percebam tudo o que ocorre quanto às verificações de acessibilidade, reduzindo as chances de diferentes resultados para um mesmo teste.

A maioria dos trabalhos descritos nesta seção foi motivada pela dificuldade de uma avaliação de acessibilidade na $W e b$, conduzindo os autores à investigação da testabilidade embutida nas WCAG 2.0. Os critérios de sucesso das WCAG 2.0 comportam-se como comandos testáveis, formando um checklist a ser usado como guia. Isso abre oportunidades para validações automáticas, embora, a validação manual ainda seja indispensável [W3C-c]. A complexidade está localizada nessa validação manual. $\mathrm{O}$ W3C, diante dessa dificuldade, sugere que o avaliador de uma página ou sítio Web seja preparado. Os autores, em [Koutsabasis et al. 2010], estendem essa sugestão, propondo que sejam incluídos organização e emprego de técnicas, no processo de avaliação. $\mathrm{O}$ autor, em [Lang 2003], vai mais além, considerando que, no relatório de uma avaliação, as falhas sejam acompanhadas de descrições para as suas soluções. Outro enfoque foi na diferença de resultados que surgiram em avaliações de uma mesma página ou sítio, efetuada por avaliadores distintos, até mesmo quando esses avaliadores tinham preparo. A expectativa é de que essas diferenças surgidas nas avaliações manuais, influenciadas por fatores subjetivos, sejam eliminadas. Outra expectativa é a de que novas metodologias tragam flexibilidade para delimitar o escopo da avaliação a um grupo particular de usuários.

\section{Plano de testes}

Neste trabalho, foram seguidas as recomendações das WCAG 2.0, que assumem o papel de um checklist, funcionando como um guia para a varredura dos critérios de sucesso pelos itens das páginas. Esse checklist serve para anotar as indicações de falhas, durante a avaliação. Com esse guia em mãos, a tarefa de avaliação é realizada por procedimentos distintos, primeiro o automático e depois o manual.

A validação automática tem a finalidade de realizar uma análise do código xHTML/HTML da página e de criticar a sintaxe da linguagem e a ausência de elementos ou atributos essenciais para acessibilidade, indicando o critério de sucesso que não está em conformidade. Um exemplo de validador automático é a ferramenta totalValidator [Total Validator].

Dando prosseguimento ao teste, após a validação automática, deve ser iniciada a validação manual. Observa-se que os itens que apresentaram falhas na validação automática já não precisam mais ser verificados nesta etapa. A validação manual desempenha um papel importante na avaliação de uma página ou sítio, sendo uma análise visual e auditiva da interface da página, que permite identificar cada item que apresente falha. Neste momento, o avaliador, com a ajuda do checklist, percorre os critérios verificando a página, por softwares leitores de tela. 
Uma vez identificada a falha, o seu registro visual pode ser realizado pelo emprego de uma ferramenta de captura e edição de tela, por exemplo, a Fireshot do Mozilla Firefox [FireShot], que permite ao avaliador apontar visualmente o erro no elemento da página; comentar; e salvar toda a imagem da página com a identificação em destaque. Essa imagem salva é útil para constituir o conjunto de imagens associadas aos itens com falha ao final de uma avaliação, no sentido de amparar o responsável pelo reparo dessas falhas. Além dessa identificação, são apontados os aspectos estruturais e semânticos do código com falha.

A primeira contribuição do presente trabalho adota a proposta de inclusão de organização e emprego de técnicas para lidar com o volume de problemas que costuma surgir, na validação manual [Koutsabasis et al. 2010]. A inclusão dessa organização, no plano de testes apresentado, foi especificada como uma ordenação das etapas de avaliação, desde a captura das telas da página ou sítio em avaliação, passando pelas etapas de validação automática, seguida da manual, e, encerrando com a organização dos resultados no relatório final.

A segunda contribuição é o acréscimo da descrição da correção da falha [Lang 2003]. Essa descrição acompanha cada indicação de item que não se encontra em conformidade com os critérios de sucesso. $\mathrm{O}$ formato dessa correção pode variar desde um acerto de código HTML/xHTML até um recurso visual ou auditivo, dependendo do tipo do atributo do item da página com a falha. Sua utilidade ocorre posteriormente ao processo de avaliação, na tarefa de reparo.

A incorporação das duas contribuições ao planejamento adotado na avaliação original, inerente aos critérios de sucesso das WCAG 2.0, representa a especificação do plano de testes, de acordo com a sequência de procedimentos apresentada, na figura 3.

Cabe notar que, após a conclusão das aplicações dos testes, três objetos que refletem os resultados devem ser gerados, como a seguir: i) relatório da análise automática, gerado quando o conteúdo é submetido à ferramenta (totalValidator); ii) arquivo com resultados do teste manual, e iii) o checklist preenchido, com os resultados dos dois testes. Todos esses três objetos retratam os quatro princípios das WCAG 2.0, porém, o avaliador deve reorganizá-los para que seja gerado um relatório único, que servirá para atestar a acessibilidade da aplicação $W e b$ ou para auxiliar o profissional responsável pelos ajustes ou reparos das falhas identificadas.

Para que o relatório unificado corresponda à finalidade de reparos e ajustes, ele deve estar organizado para possibilitar a identificação dos seus seguintes itens: os critérios de sucesso das WCAG 2.0 associados às falhas encontradas; o nível de conformidade relacionado ao critério; e uma descrição sucinta da falha.

Nas figuras 4 e 5, são apresentados trechos do relatório gerado na avaliação de acessibilidade, em que foi adotado este plano de testes. O relatório completo encontra-se em [Nardi and Sacramento 2011]. Para auxiliar a identificação, a imagem ou o código com a falha estão destacados em vermelho e a sugestão de ajuste está destacada em azul. Se a representação da falha por código fica inviável, é necessário capturar algumas telas, para ilustrar os problemas nos critérios de sucesso. Por fim, deve ser anotado o procedimento aconselhado para a correção, seguindo os padrões do $\mathrm{W} 3 \mathrm{C}$, relacionados a linguagens para desenvolvimento HTML/xHTML e CSS. Maiores esclarecimentos devem ser adicionados à descrição da correção da falha, caso seja importante e útil. 
1. Capturar telas da página do sítio

1.1 Salvar em diretório print no formato JPG, nomeando com o seguinte padrão:

AAAAMMDD_NomeDaSeçãoDoSítio_NomeDaSubseção(se houver).JPG

2. Analisar a seção do site dividida em princípios e diretrizes do WCAG 2.0

2.1 Aplicar validador automático de acessibilidade, totalValidator, nos critérios de sucesso e suas recomendações

2.1.1 Para cada critério de sucesso que não esteja em conformidade, registrar problema encontrado, indicando: o critério de sucesso, descrição sucinta, código xHTML/HTML incorreto destacado em vermelho e negrito e código xHTML/HTML de uma possível solução, destacada em azul e negrito;

2.1.2Para cada critério de sucesso que não esteja em conformidade, indicar no documento "WCAG 2.0 checklist" que este não passou nos testes automáticos.

2.2 Aplicar validação manual a partir do documento "WCAG 2.0 Checklist"

2.2.1 Para cada critério de sucesso que esteja em conformidade, marcar no checklist,

2.2.2Para cada recomendação que não esteja em conformidade, registrar problema encontrado, indicando: o critério de sucesso, descrição sucinta, código xHTML/HTML incorreto, destacado em vermelho e negrito e/ou destaque da falha com contorno em vermelho rotulado com o critério de sucesso correspondente, na interface da seção capturada, e código xHTML/HTML indicando uma possível solução destacada em azul e negrito e/ou em interface capturada, com contorno(s) desenhado(s) em verde rotulado com o critério de sucesso correspondente;

[Os arquivos de interface gerados na análise devem estar em formato JPG e ser nomeados com o seguinte padrão:AAAAMMDD_NomeDaSeçãoDoSítio_NomeDaSubseção(se houver)_I ou C.JPG]

3. Organizar resultados obtidos em relatório com o seguinte formato:

Nome do Princípio

Citação do princípio

Número da Diretriz

Citação da diretriz.

Número do critério de sucesso

Citação do critério de sucesso verificado.

A) Recomendações que não apresentaram falhas

Citação do item no checklist que não apresentou falha, se houver.

B) Recomendações que apresentaram falhas

B.1) Avaliação Automática:

Citação do item no checklist que apresentou falha, se houver.

Descrição sucinta da falha.

Código HTML incorreto:

Número da linha | Código com problema indicado em vermelho.

Código HTML correto:

Número da linha | Código com sugestão de correção indicado em azul.

Descrição do procedimento adotado.

Observação.

B.2) Avaliação Manual:

Citação do item no checklist que apresentou falha, se houver.

Descrição sucinta da falha.

Código HTML incorreto:

Número da linha | Código com problema indicado em vermelho Ou

Arquivo de imagem ilustrando o problema:

Imagem da página capturada destacando a falha com contorno em vermelho rotulado com o critério de sucesso

correspondente

Código HTML correto:

Número da linha | Código com sugestão de correção indicado em azul. Ou

Arquivo de imagem ilustrando a solução:

Imagem da página capturada com uma possível solução com contorno(s) desenhado(s) em verde rotulado com o critério de sucesso correspondente;

Descrição do procedimento adotado.

Observação.

C) Recomendações que não se aplicaram a página analisada

Citação do item no checklist que não se aplicou, se houver.

Figura 3. Plano de testes 


\section{Critério de sucesso 1.1.1}

[22] "Conteúdo Não Textual: Todo o conteúdo não textual que é apresentado ao usuário tem uma alternativa em texto que serve um propósito equivalente, exceto para as situações indicadas abaixo. (Nivel A)"

\section{A)Recomendações que não apresentaram falhas}

[32] "Imagens apenas decorativas, utilizadas apenas para formatação visual ou imagens onde o conteúdo é transmitido em texto são fornecidos textos alternativos vazios (alt = "”) ou são implementadas como plano de fundo CSS. Todas as imagens com link possuem texto alternativo descritiva."

\section{B)Recomendações que apresentaram falhas}

\section{B.1) Avaliação Automática:}

Nenhuma

\section{B.2) Avaliação Manual:}

[32] "Todas as imagens, botões de imagem em formulários, e regiões de mapas de imagens possuem texto alternativo equivalente, apropriado". Descrição sucinta da falha: A imagem não possui texto alternativo apropriado.

Códigos HTML incorretos:

$\begin{aligned} & \text { (1) } 93<\text { <img } \\ & \text { alt="Home"/> }\end{aligned}$
src=http://www.bitwebjr.com.br/malice/sites/default/files/malice.png

(2) $114<$ img src="sites/ime.png" alt="http://www.ime.uerj.br/" width="70px" heigth="80px"/>

(..)

Códigos HTML corretos:

(1) 93 <img src="http://www.bitwebjr.com.br/malice/sites/default/files/malice.png"
alt="Fotografia da professora Maria Alice com flores ao fundo"/>

(2) $114<$ img src="sites/ime.png" alt="Logotipo do Instituto de Matemática e Estatística IME - UERJ" width="70px" heigth="80px"/>

\section{(..)}

Procedimento: Identificamos no código fonte da página, os elementos de imagem e avaliamos se o texto alternativo descrito no atributo 'alt' é equivalente e apropriado à imagem em referência. Percebemos nas imagens (1), (4) e (7), que o texto alternativo correspondia ao destino do link, nas imagens (2) e (3) correspondem ao link do sítio das instituições referenciadas e nas imagens (8) e (9) os textos alternativos não correspondem ao que as imagens representam, sendo idênticas ao atributo 'title'.

Observação: Verifica-se que há uma redundância dos elementos <img>, nos itens (2) e (3) com os itens (5) e (6), i.e, a mesma descrição de texto alternativo, bem como as imagens. Portanto não é necessária uma segunda referência.

\section{C)Recomendações que não se aplicaram à página analisada}

[32] "Alternativas textuais equivalentes a imagens complexas são fornecidas no contexto ou em página separada (vinculados e / ou referenciadas através longdesc); Botões de formulário possuem um valor descritivo; Entradas de formulário (input) possuem etiquetas (label) associadas, ou se etiquetas não puderem ser usadas, um atributo título (title) descritivo; Multimídia incorporado é identificado através de textos acessiveis; Quadros (frames) são intitulados apropriadamente."

Figura 4. Trecho do relatório - item que não se encontra em conformidade com o critério de sucesso 1.1.1. 


\section{Critério de sucesso 3.1.2}

[22] "Linguagem das Partes: A linguagem humana de cada passagem ou frase do conteúdo pode ser determinada de forma programática, exceto para os nomes próprios, os termos técnicos, palavras de idioma indeterminado e palavras ou frases que se tornaram parte do vernáculo do texto imediatamente circundante. (Nível AA)".

A) Recomendações que não apresentaram falhas

Nenhuma

B) Recomendações que apresentaram falhas

B.1) Avaliação Automática:

Nenhuma

B.2) Avaliação Manual:

[32] "Quando necessário, o idioma das seções de conteúdo que sejam diferentes do idioma principal são identificados, por exemplo, usando o atributo lang (<blockquote lang="es")>”.

Descrição sucinta: Palavras em outros idiomas diferentes do idioma principal da página, devem estar destacadas com indicação do idioma da palavra, para que usuários que façam uso de leitores de tela, ouçam a fonética correta da palavra.

Exemplo: O texto Skip to main content, termo presente logo no início do site, sem a indicação de que se trata de uma expressão em idioma inglês, teria a fonética: [Skip tu main contenti]



Código HTML incorreto:

(1) $50<$ div id="skip-link">

$51<$ a href="\#main-content" class="element-invisible element-focusable" $>$ Skip to main content $</ a>$

$52</ \operatorname{div}>$

(...)

Código HTML correto:

$$
\begin{aligned}
& (1) 50<\text { div id="skip-link" }> \\
& 51<\text { a href="\#main-content" class="element-invisible element-focusable" }><\text { span } \\
& \text { lang="en" }>\text { Skip to main content }</ \text { span }></ \text { a }> \\
& 52<\text { div }> \\
& (\ldots)
\end{aligned}
$$

Procedimento: Em cada expressão em idioma diferente do padrão da página foi necessário inserir um elemento de linha $<$ span $>$ (coringa) com atributo "lang", indicando sua linguagem nativa. Desta forma, o sentido semântico correto é atribuído à palavra.

*Obs.: Fonética extraída de Longman Dictionary of Contemporary English - $1^{\circ}$ Edição - Editora Longman 
$\mathrm{Na}$ diagramação desse relatório, para cada um dos princípios, as definições de diretrizes e de seus critérios de sucesso constantes da WCAG 2.0 devem estar dispostas de forma organizada.

Em uma avaliação apoiada por este plano de testes, os resultados mostraram que 30 critérios de sucesso não se aplicaram ao conteúdo submetido ou foram inviáveis. Assim sendo, foram considerados apenas 31 critérios de sucesso.

A ordenação adicionada ao processo, explícita neste plano de testes, facilitou o trabalho de avaliação, mesmo com um número de indicações significativo, em que foram apontados 13 critérios de sucesso em conformidade, em 22 itens, e 18 com falhas, em 43 itens avaliados. Os itens com falha foram acompanhados das correções, que são úteis nas tarefas de reparo, cuja simplificação encoraja esse trabalho, viabilizando o alcance da acessibilidade na página avaliada.

A etapa de validação manual transcorreu com cuidado para que aspectos subjetivos não influenciassem o resultado da avaliação.

\section{Conclusões}

Este trabalho teve como motivação principal o aprimoramento da acessibilidade na $W e b$ e descreveu um plano de testes apresentado como extensão do planejamento de avaliação de acessibilidade, inerente aos critérios de sucesso das WCAG 2.0.

Um objetivo deste trabalho foi lidar com o volume de problemas que costuma surgir na etapa de validação manual, para tanto o plano de testes especifica uma ordenação das etapas de avaliação e organiza os resultados no relatório final.

O outro objetivo foi a inclusão de sugestões de correção para as falhas apresentadas. Dessa forma o plano de testes acrescenta a descrição da correção de falha para cada item que não apresenta conformidade com os critérios de sucesso das WCAG 2.0 .

Para os trabalhos futuros, é pretendida a investigação nas seguintes questões: i) uma seleção de critérios de sucesso mais apropriada aos objetivos de uma avaliação, que pode diminuir a complexidade nesse processo; e ii) o desenvolvimento de ferramentas automáticas que substituam cada vez mais a validação manual, que pode colaborar com a redução dos esforços despendidos pelos avaliadores e com resultados mais precisos.

\section{References}

Alonso, F., Fuertes, J., González A. and Martínez L. (2010). "On the testability of WCAG 2.0 for beginners". In: W4A '10 - The 7th International Cross-Disciplinary Conference on Web Accessibility - SESSION: Testing and validation, Raleigh, North Carolina, USA.

Bailey, C. and Pearson, E. (2010). "An educational tool to support the accessibility evaluation process". In: W4A '10 - The 7th International Cross-Disciplinary Conference on Web Accessibility - SESSION: Testing and validation, Raleigh, North Carolina, USA.

Bechara, E. (2009). "Recomendações de Acessibilidade para Conteúdo Web (WCAG) 2.0”. Disponível em: <http://www.ilearn.com.br/TR/WCAG20/> 
Brajnik, G., Yesilada, Y. and Harper, S. (2010). "Testability and Validity of WCAG 2.0: The Expertise Effect". In: ASSETS'10 - The 12th International ACM SIGACCESS Conference on Computers and Accessibility, Orlando, USA.

CRPD - United Nations. (2008). "Convention on the Rights of Persons with Disabilities”. Headquartes, New York. Disponível em: http://www.un.org/disabilities/convention/conventionfull.shtml

Decreto 5.296. "Decreto n 5.296 - regulamenta Leis 10.048 e 10.098, pertinentes à acessibilidade." (2004). Disponível em:

<http://www.planalto.gov.br/ccivil_03/_ato2004-2006/2004/decreto/d5296.htm>

Dias, C. (2007) "Usabilidade na Web: Criando portais mais acessíveis", Alta Books Ltda, 2. ed, 296 p. Rio de Janeiro.

DOSVOX. "Sistema Operacional DOSVOX”. Núcleo de Computação Eletrônica da Universidade Federal do Rio de Janeiro (UFRJ). Disponível em:

$<$ http://intervox.nce.ufrj.br/dosvox $>$

e-MAG. "Modelo de Acessibilidade de Governo Eletrônico". (2005). Departamento de Governo Eletrônico. Disponível em: $<$ http://www.governoeletronico.gov.br/acoes-eprojetos/e-MAG>

Fernandes, N., Lopes, R. and Carriço, L. (2011) "On Web accessibility evaluation environments". In W4A '11: Proceedings of the International Cross-Disciplinary Conference on Web Accessibility, Hyderabad, India.

FireShot. Suslikov, E. "FireShot Screenshot tool - Capture and Annotate".Disponível em: <https://addons.mozilla.org/ptbr/firefox/addon/fireshot $>$

Gay, G. and Qi Li, C. (2010) "AChecker: open, interactive, customizable, Web accessibility checking". In: W4A '10 - The 7th International Cross-Disciplinary Conference on Web Accessibility - SESSION: Testing and validation, Raleigh, North Carolina, USA.

Gil, M. (2006). “Acessibilidade, Inclusão Social e Desenho Universal: Tudo a Ver”. Disponível em: <http://www.bengalalegal.com/martagil>

Henry, S. (2006). "Understanding Web Accessibility", Web Accessibility: Web Standards and Regulatory Compliance, Springer-Verlag New York, Inc., 2006, pp. $1-51$.

IDEA United States. (2005). The Individuals With Disabilities Education Act (IDEA), 20 U.S.C. Chapter 33 - Section 1401. Disponível em: $<$ http://uscode.house.gov/uscode-cgi/>

JAWS. "JAWS for Windows Screen Reading Software”. Freedom Scientific, Inc. Disponível em: <http://www.freedomscientific.com/products/fs/jaws-productpage.asp $>$

Koutsabasis, P., Vlachogiannis, E. and Darzentas, J. (2010). "Beyond Specifications: Towards a Practical Methodology for Evaluating Web Accessibility". Journal of Usability Studies. Vol. 5, 157-171. 
Lang, T. (2003) "Comparing website accessibility evaluation methods and learnings from usability evaluation methods" disponível em: http://www.peakusability.com.au/about-us/pdf/website_accessibility.pdf

Lawrence, S. and Giles, C. (2000). "Accessibility of information on the Web", In Magazine intelligence, ACM, New York, USA, vol. 11, Spring.

Lazar, J., Dudley-Sponaugle A. and Greenidge, K. (2004). "Improving Web accessibility: a study of webmaster perceptions", In: Computers in Human Behavior, Elsevier, vol. 20, pp. 269-288

Melo, A., Baranauskas, M. and Bonilha, F. (2004) "Avaliação de Acessibilidade na Web com a Participação do Usuário", In: Anais do VI Simpósio de Fatores Humanos em Sistemas Computacionais, Curitiba, Brasil.

Nardi, L. and Sacramento, C. (2011). “Avaliação da acessibilidade de uma página Web de acordo com as WCAG 2.0", Trabalho de Conclusão de Curso, Departamento de Informática e Ciência da Computação, IME, UERJ, Rio de Janeiro, Brasil.

NV Acces. "NonVisual Desktop Access". NV Access. Disponível em: $<$ http://www.nvda-project.org/wiki/AccessingAndRunningSourceCode $>$

Sierkowski, B. (2002). "Achieving Web Accessibility", In: Proceedings of the 30th annual ACM SIGUCCS conference on User services, Rhode Island, USA.

Slatin, M. and Rush, S. (2003). "Maximum accessibility: Making your Web sites more usable for everyone". Addison-Wesley Publishing. Boston.

Total Validator.“Total Validator Home Page”. Total Validator. Disponívem em: < http://www.totalvalidator.com

UDI-a. Universal Design Institute. "What is Universal Design?". Disponível em: $<$ http://www.udinstitute.org/whatisud.php $>$

UDI-b. Universal Design Institute. (1997). "Principles of Universal Design". Disponível em: <http://www.udinstitute.org/principles.php>

Vieira, A. (2010). "Dimensões e Características da Web Brasileira”, In: Comitê Gestor da Internet no Brasil - CGI.br e Núcleo de Informação e Coordenação do Ponto BR - NIC.br. Disponível em: http://www.cgi.br/publicacoes/pesquisas/govbr/cgibrnicbr-censoweb-govbr-2010.pdf

Virtual Vision. "Virtual Vision: Acessibilidade para deficientes visuais". Micro Power. Disponível em: http://www.virtualvision.com.br

W3C-a. World Wide Web Consortium, Laboratory for Computer Science, Massachusetts Institute of Technology, USA. Disponível em: $<$ http://www.w3.org $>$

W3C-b. (1999). “Web Content Accessibility Guidelines 1.0”. Disponível em: $<$ http://www.w3.org/TR/WCAG10>

W3C-c. (2008). “Web Content Accessibility Guidelines (WCAG) 2.0”. Disponível em: $<$ http://www.w3.org/TR/WCAG>

W3C-d. "Web Accessibility Evaluation Tools: Overview". Disponível em: $<$ http://www.w3.org/WAI/ER/tools $>$ 
W3C-e. "Standards, Web Design and Applications, Accessibility". Disponível em: $<$ http://www.w3.org/standards/webdesign/accessibility>

W3C-f. "Web Accessibility Initiative”. Disponível em: http://www.w3.org/WAI

WCAG 2.0-pt. "Directrizes de Acessibilidade para o Conteúdo da Web (WCAG) 2.0". Acesso UMIC. Disponível em: <http:/www.acesso.umic.pt/w3/TR/WCAG20>

WebAIM. Web Accessibility in Mind. "WebAIM's WCAG 2.0 Checklist”. Disponível em: <http://webaim.org/standards/wcag/checklist> 